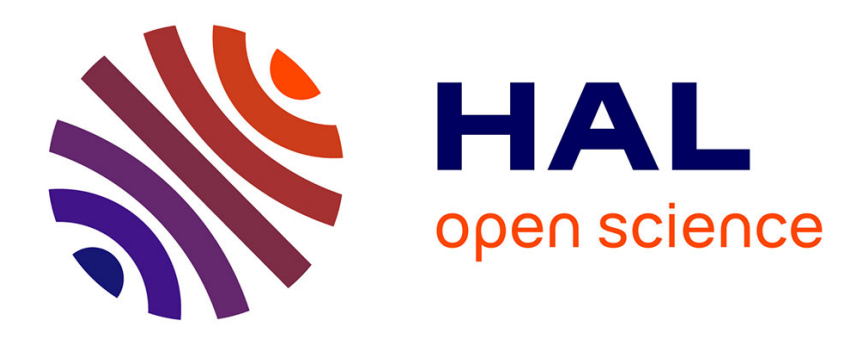

\title{
Les oiseaux nicheurs en Grésigne (Tarn)
}

Bernard Alet

\section{To cite this version:}

Bernard Alet. Les oiseaux nicheurs en Grésigne (Tarn). Revue Géographique des Pyrénées et du Sud-Ouest, 1984, 55 (3), pp.392-395. 10.3406/rgpso.1984.4614 . hal-02624021

\section{HAL Id: hal-02624021 \\ https://hal-univ-tlse2.archives-ouvertes.fr/hal-02624021}

Submitted on 26 May 2020

HAL is a multi-disciplinary open access archive for the deposit and dissemination of scientific research documents, whether they are published or not. The documents may come from teaching and research institutions in France or abroad, or from public or private research centers.
L'archive ouverte pluridisciplinaire HAL, est destinée au dépôt et à la diffusion de documents scientifiques de niveau recherche, publiés ou non, émanant des établissements d'enseignement et de recherche français ou étrangers, des laboratoires publics ou privés. 


\section{Les oiseaux nicheurs en Grésigne (Tarn)}

\section{Bernard Alet}

\section{Citer ce document / Cite this document :}

Alet Bernard. Les oiseaux nicheurs en Grésigne (Tarn). In: Revue géographique des Pyrénées et du Sud-Ouest, tome 55, fascicule 3, 1984. pp. 392-395;

doi : https://doi.org/10.3406/rgpso.1984.4614

https://www.persee.fr/doc/rgpso_0035-3221_1984_num_55_3_4614

Fichier pdf généré le 06/04/2018 
Mobiles et sensibles, souvent discrets dans les feuillages, les oiseaux sont difficiles à situer et à raisonner dans la mosaïque changeante des paysages. L'intérêt de leur étude n'en est que plus évident. Longtemps réduite à des inventaires subjectifs, l'ornithologic fait tous les jours des progrès essentiels: méthodes d'échantillonnage, analyses quantitatives des données, travaux de génétique des populations, modélisations. Cependant le rapport de l'oiseau à l'espace et au temps demeure mal maîtrisć et la cartographie ornithologique, synchronique ou diachronique, en particulier à grande ćchelle, reste sinon à inventer, du moins à organiser. Le géographe y trouve à la fois une place, d'ailleurs reconnue, et une raison d'être. A deux conditions : qu'il soit porteur de connaissances spécifiques et de méthodes opérationnelles et qu'il participe à une démarche transdisciplinaire. En fait, la motivation du géographe doit être plus large encore, dans la perspective d'un redéploiement de sa discipline. D'abord, la biogéographie doit être perçue non seulement comme une phytogéographie mais aussi comme une zoogéographie. Ensuite, il s'agit de démontrer que la géographie physique n'a pas pour seul interlocuteur la géographic humaine ou sociale mais l'ensemble des sciences de la nature et de la société, et en particulier l'écologie et l'histoire (1).

Par la diversité de leurs paysages et la complexité de leur histoire, les pays de Grésigne (Tarn) constituent un terrain d'excrcice approprié. Le massif forestier de Grésigne (500 m d'altitude à Pech-Aguze) s'élève au contact de trois grands domaines naturels: les Causses du Quercy, le Scigala rouergat et tarnais, les coteaux molassiques du Bassin Aquitain oriental. Sa position biogéographique, sa situation d'éperon avancé du Massif Central vers le Sud-Ouest aquitain font de ce massif une entité géographique où interfèrent des tendances très contrastées; d'où l'originalité et la diversité de l'avifaune. Enfin, si de nombreux travaux de phytogéographie y ont été réalisés (2), il n'existe par contre aucune étude ornithologique approfondie, a fortiori aucun essai d'ornithogéographie.

Au point de départ de cette étude se trouve l'analyse intégrée des milieux naturels de Grésigne (3) conduite selon la méthode des géosystèmes, c'est-à-

1. Aurt B., L'avifaune dans les géosystèmes de Grésigne (Tarn). Essai de cartographie ornithogéographique. Doctorat de 3" cycle Géographie, Toulouse, 1984, 278 p., 51 fig., biblio.

2. GiYriRd C., (1970), Phytogéographie de la région de Grésigne (Tarn). Toulouse, CRDP, Documents d'écologie végétale et forestière, $166 \mathrm{p}$. Di.P.Y B., (1979), Contribution à l'étude stationnelle d'une forêt de chênes: le massif domanial de Grésigne (Tarn). Thèse $3^{*}$ cycle Botanique et Biogéographie, Toulouse.

3. Alet B., Andrès M., Bertix M., Pichei. C., et Viguié B., (1980), Pays et paysages grésignols. Mémoire de Maîtrise de Géographie, Toulouse, 200 p. + $\mathrm{XX}$ p. +6 cartes hors-texte en couleur au 1/25000". All:T B. et Bl:RTIN M., (1981), Les géosystèmes de (irésigne ('Tarn). Mémoire DEA de Géographie, Toulouse, 120 p. +1 carte hors-texte en couleur au $1 / 25000^{\circ}$. 
dire, en gros, par la caractérisation et l'organisation spatiaie, à leurs échelles respectives, des diflérentes unités territoriales: géosystèmes, géofaciès, géotopes (4). La méthode a été allinćc et mise en adéquation avec le thème ornithologique: définttion des géohorizons à l'intéricur de chaque géofaciès pour déterminer les stratégies verticales d'occupation de l'espace par les différentes espéces d'oiseaux aux différentes périodes de l'annéc; construction d'un ensemble de sept cartes superposables à $1 / 25000$ dont la carte des géosystemes et surtout trois cartes historiques: utilisation de l'espace au XIX" siècle (d'après les relevés cadástraux de 1815-1835); utilisation de l'espace au XX" siècle (d'après les photographies aćriennes et les relevés de terrain); entin, carte de l'évolution des paysages du XIX" au XX" siècles.

L'inventaire tient compte à la fois de la méthode d'échantillonnage de l'avifaune par points d'écoute, fondéc sur la reconnaissance du chant des oiseaux qui caractérise chaque espèce, et de la méthode géosystémique. La carte des géosystemes, arec son dicoupage en géofaciès, est le point de départ des relevés d'avifaune. On échantillonne, d'une part, le centre de chaque géofaciès et, d'autre part, les zones de contact entre les différentes unités territoriales. La méthode prend done en compte l'hétérogénéité des milieux et leurs écotones (rones de transition entre deux milieux). Un quadrillage préalable et au hasard du terrain est done inutile. L'échantillonnage porte sur les 49 géofaciès cléfinis et cartographiés (carte des géosystèmes), ce qui représente par conséquent une cinquantaine de points d'écoute réalisés au centre de chaque géofacios au printemps 1982. Afin de tester la méthode, des relevés supplémentaires ont été effectués en divers points d'un même géofaciès. Il a fallu également procéder à un échantillonnage des zones de contact les plus caractéristiques. Ces écotones sont de nature très diverse mais peuvent être regroupés selon l'importance de la discontinuité spatiale et selon le sens et le pas du temps de leur évolution (depuis le milieu du XIX" siècle, depuis 30 ans...).

Après un regroupement physionomique des géofaciès, on obtient 21 peuplements d'oiseaux nicheurs qui rassemblent les 102 espèces présentes en Grésigne au printemps 1982. Les oiseaux utilisent la régétation non seulement en tant que support mais aussi en tant que source de nourriture, laquelle varie au cours des saisons en fonction du rythme biologique des végétaux, leur fructification par exemple. La carte générale des peuplements d'oiseaux représente la répartition spatiale des oiseaux les plus caractéristiques, dans la mesure où chaque espèce développe sa propre stratégie d'utilisation de l'espace non seulement horizontalement mais aussi verticalement (depuis le sol et même le sous-sol jusqu'à l'espace aérien, en passant par les différentes strates de végétation).

Prenons le cas de l'avifaune forestière avec le peuplement d'oiseaux de la vieille futaie de hêtres et de chênes rouvres sur les grands versants humides en forêt domaniale. Les espèces inféodées à ce milieu sont le Pouillot siffleur (Phylloscopus sibilatrix), la Bécasse (Scolopax rusti-

4. Bertrand G. et al., (1978), Géosystème et aménagement, RGPSO, 49, 2. 
cola), les trois pics du genre Dendrocopos...; ces trois pics ont leur propre stratégie d'utilisation de l'arbre: alors que le Pic épeiche (Dendrocopos major) se tient davantage à faible hauteur sur les troncs et les branches maîtresses, le Pic mar (Dendrocopos medius) et le Pic Epeichette (Dendrocopos minor) utilisent les strates arborescente et arborée, le dernier exploitant les extrémités des branches les plus fines.

Les peuplements d'oiseaux ont été regroupés et analysés au sein de quatre grandes avifaunes correspondant aux quatre grandes unités territoriales mises en évidence par la carte des géosystèmes : causses en voie d'abandon, complexe forestier, complexe agricole et enfin, complexe bocager aux écotones multiples. A ces quatre avifaunes largement répandues, il faut ajouter différentes avifaunes spatialement plus limitées, liées à des habitats marginaux, ponctuels (bourgs, hameaux, mares...) ou linéaires (ripisylves, cours d'eau, parois calcaires...).

Il est intéressant, enfin, d'évoquer le contraste biogéographique qui existe entre deux espèces d'oiseaux nicheurs de la mème famille (Sylviidés), séparées l'une de l'autre par une distance d'environ $1500 \mathrm{~m}$ seulement: il s'agit, d'une part, du Pouillot siffleur (Phylloscopus Sibilatrix), espèce "septentrionale» inféodée à la chênaic-hètraie de Montoulieu en forêt domaniale, et, d'autre part, de la Fauvette passerinette (Sylvia cantillans), espèce laté-méditerranéenne inféodée au taillis clair de chênes verts et pubescents du plateau calcaire de Larroque (fig. 1). Cette opposition, calquée sur celle des géosystèmes et tout particulièrement sur celle de la végćtation, souligne la position charnière du massif de Grésigne.

L'analyse spatio-temporelle autorise, avec quelques réserves, l'hypothèse de scénarios ou "modèles" d'évolution depuis le milicu du XIX" siècle parfois. En effet, les peuplements d'oiseaux se modifient constamment en fonction de l'action anthropique: svlviculture en forei domaniale, plantation de résineux et de peupliers, changement d'assiette et de technique de culture du vignoble du Gaillacois, remplacement de cultures par d'autres, remembrement de la vallée de la Vère, etc. Dans ces scénarios, les oiseaux sont utilisés comme indicateurs de l'évolution des milieux, compte tenu des diverses stratégies adaptatives (colonisation, disparition, adaptation).

C'est, par exemple, le cas de l'avifaune des friches et des causses en voie d'abandon. L'exode rural depuis la lin du XIX" siècle et surtout depuis la seconde guerre mondiale entraîne la "fermeture » progressive du couvert végétal. Au stade de la pelouse-lande à Brachypodium pinnatum correspond un peuplement d'oiseaux particulier avec notamment l'Alouette lulu (Lullula arborea), le Pipit rousseline (Anthus campestris), le Traquet pâtre (Saxicola torquata). Au stade suivant, celui de la lande à fruticées avec Genévrier et Prunus, apparaissent la Linotte (Acanthis cannabina), la Fauvette grisette (Sylvia communis), l'Hypolaïs polyglotte (Hippolaïs polyglotta). Enfin, dans la lande arborée à chêne pubescent, ces espèces disparaissent progressivement et sont remplacées par lc Pipit des arbres (Anthus trivialis), le Pouillot de Bonelli (Phylloscopus bonelli).

L'analyse géosystémique de l'avifaune ne permet pas seulement de replacer les peuplements d'oiseaux dans l'espace et le temps. Elle précise 


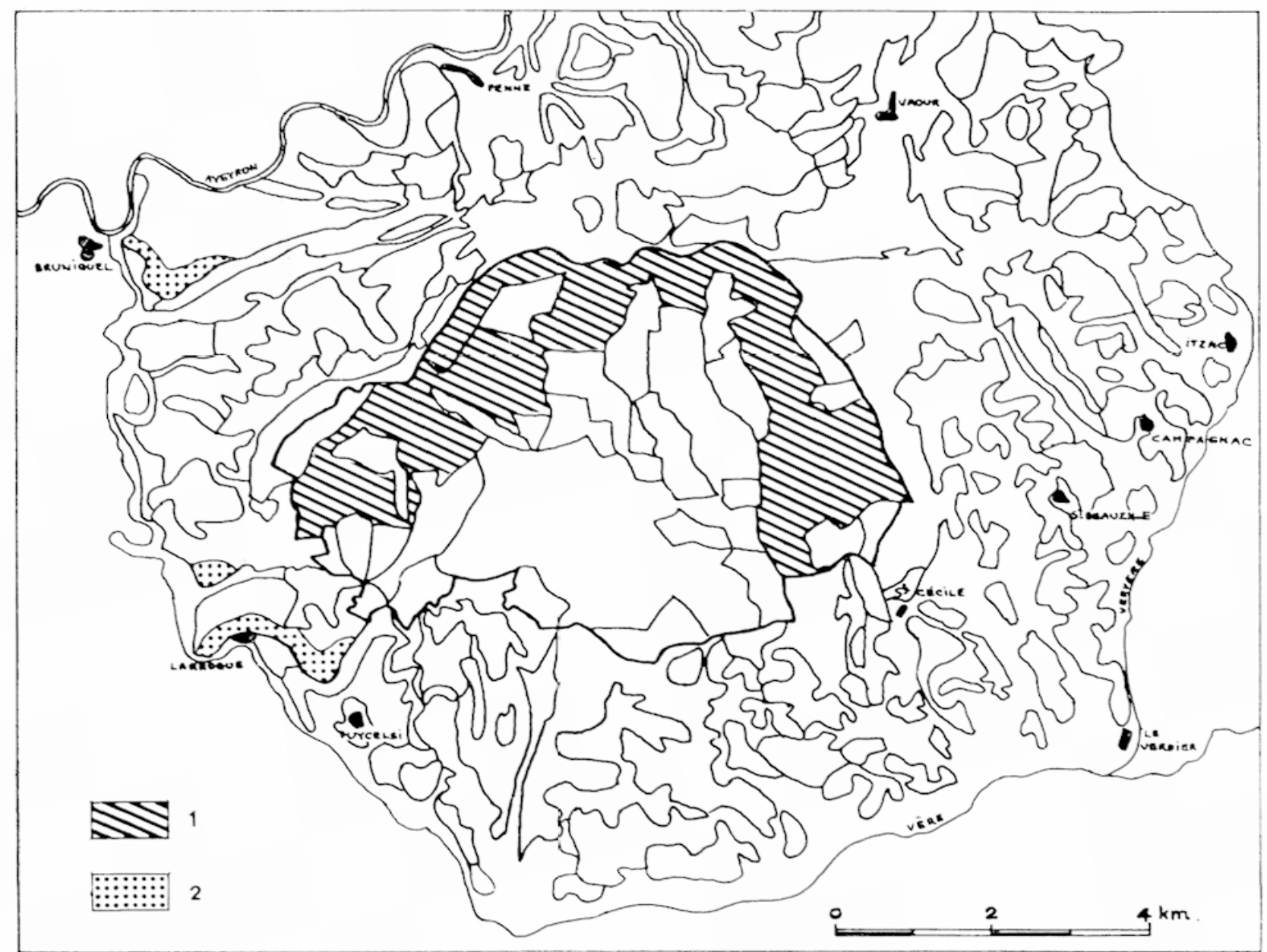

FIG. 1

\section{Localisation de deux espèces d'oiseaux nicheurs}

1. Pouillot siffleur. - 2. Faurette passerinette.

aussi certains états ou évolutions des milieux. La cartographie ornithogéographique à grande échelle peu donc se révéler utile dans la réalisation de plans de gestion et d'aménagement de l'espace. En resituant les différents peuplements d'oiscaux et même certaines espèces dans leur espace et dans leur histoire, compte tenu de leur phénologie, on peut les considérer comme des bio-indicateurs aussi fiables que sensibles.

La deuxième étape de ce travail, aujourd'hui en cours, consiste à préciser et à généraliser la méthode testée en Grésigne grâce au traitement statistique et à la modélisation de systèmes spatiaux ornithologiques. La géographie et l'ornithologie (et plus généralement la zoologie) ont encore un long chemin à faire ensemble.

Bernard Alet. 